\title{
EVALUACIÓN DE LA ESTIMACIÓN DE METRADOS PARA LOS COSTOS DE LA PARTIDA DE ARQUITECTURA DE UNA OBRA RETAIL EN LIMA EN EL 2019 CON LA IMPLEMENTACIÓN BIM
}

Pablo Medina_Choccetoy, Nataly Salomon_Arce, Rosmery Gómez_Minaya

\section{RESUMEN}

En las obras retail del tipo llave en mano y ejecutados con el método convencional, generan diversos errores, retrasos, sobrecostos en las obras, revisiones en la etapa de ejecución y con ello demoras en la estimación de metrados, obteniendo una baja eficiencia e incremento de costos, tiempo y mala calidad. Se determinó la eficiencia que logra la implementación de BIM en la partida de arquitectura de una obra retail, evaluando dos retail ejecutados con el método convencional y dos retail implementando BIM, estimando los metrados e identificando la excedencia o déficit de la cuantificación de metrados, el tiempo y costo que toma en realizar una revisión. Se obtuvo la eficiencia, mediante la evaluación de tres indicadores (costo, tiempo y calidad), generando escalas con puntuaciones de 0 a 5 para cada indicador y una tabla de porcentajes de eficiencia, determinando rangos de $0 \%$ a $100 \%$, representando gráficamente mediante el Benchmark la puntuación de indicadores de cada retail. La implementación BIM en la estimación de costos en la partida de arquitectura si resulta ser eficiente, ya que en los dos retail con BIM se obtuvieron un 95\% de eficiencia, y en los dos retail sin BIM alcanzaron valores de $43 \%$ y $33 \%$. También, se implementó dos diagramas de flujo para la comparación de ambos métodos (tradicional y BIM), demostrando que existe una diferencia de 8 días entre ambos, siendo el método BIM el más eficiente, ya que solo contó con 3 días para realizar todo el proceso de metrados y revisión.

Palabras Clave: BIM, Calidad, Tiempo, Costo, Cuantificación, Metrados.

DOI: 10.23881 idupbo.020.1-12i 